\title{
Determinação amperométrica de tiocarbamida em meio aquoso utilizando um eletrodo modificado com ferricianeto de cobre
}

\author{
Edervaldo Buffon (PG) $)^{1-2}$; Maria de Lourdes S. Vasconcellos $(P G)^{1-3^{*}}$; \\ Demetrius Profeti $(P Q)^{1}$; Luciene P.R. Profeti $(P Q)^{1}$ \\ ${ }^{1}$ Departamento de Química e Física, Universidade Federal do Espírito Santo, Alegre, ES, Brasil.
}

${ }^{2}$ Programa de Pós-Graduação em Química, Departamento de Química Analítica, Instituto de Química de Araraquara, Universidade Estadual Paulista Júlio de Mesquita Filho, Araraquara, SP, Brasil.

${ }^{3}$ Programa de Pós-Graduação em Química, Universidade Federal do Espírito Santo, Vitória, ES, Brasil

* e-mail: lourdes-vasconcellos@hotmail.com

\begin{abstract}
Resumo
Neste trabalho, um eletrodo de carbono vítreo (GCE) modificado com ferricianeto de cobre (CuHCF) foi aplicado na determinação de tiocarbamida em meio aquoso, uma vez que a essa molécula é o produto da degradação dos compostos sulfurados nos recursos hídricos em geral. $O$ eletrodo modificado foi preparado eletroquimicamente por voltametria cíclica em uma solução contendo ferricianeto, $\mathrm{Cu}$ (II) e nitrato de potássio. O CuHCF mostrou boa eletroatividade na presença da molécula de tiocarbamida e uma pronunciada corrente de pico anódica foi observada no voltamograma cíclico no potencial de $0,69 \mathrm{~V}$ $(\mathrm{Ag} / \mathrm{AgCl})$. A resposta analítica, investigada por cronoamperometria, apresentou um comportamento linear no intervalo de concentração de $5.0 \times 10^{-4}$ a $30.0 \times 10^{-4} \mathrm{~mol} \mathrm{~L}^{-1}(r=0,99997)$ de tiocarbamida, com limite de detecção (LOD) de $1,6 \times 10^{-5} \mathrm{~mol} \mathrm{~L}^{-1}$. Estes resultados mostram a promissora aplicação deste eletrodo para a determinação de tiocarbamida em amostras reais.
\end{abstract}

\begin{abstract}
In this work a, glassy carbon electrode (GCE) modified with copper ferricyanide (CUHCF) was applied in determination of thiocarbamide in aqueous medium, since this molecule is a product of degradation of sulfur compounds in water resources. The modified electrode was electrochemically prepared by cyclic voltammetry from a solution containing ferricyanide, $\mathrm{Cu}(\mathrm{II})$, and potassium nitrate. The CuHCF modified electrode has shown good electroactivity in the presence of thiocarbamide, and a pronounced anodic peak current was observed in cyclicvoltammogram at $0.69 \mathrm{~V}(\mathrm{Ag} / \mathrm{AgCl})$. The analytical performance investigated by chronoamperometry presented a linear behavior over the thiocarbamide concentration in the range $5.0 \times 10^{-4}-30.0 \times 10^{-4} \mathrm{~mol} \mathrm{~L}^{-1}$, with a correlation coefficient ( $r$ ) of 0.99997 , and a limit of detection (LOD) of $1,6 \times 10^{-5} \mathrm{~mol} \mathrm{~L}^{-1}$. These results show the promising applicability of this electrode for the determination of thiocarbamide in real samples.
\end{abstract}

Keywords (Palavras chaves): Eletrodo Modificado, Ferricianeto de Cobre e Tiocarbamida.

\section{Introdução}

Atualmente há uma grande preocupação entre os pesquisadores com os compostos que possuem grupos sulfetos em sua constituição, uma vez que estes são bioacumulativos e tóxicos para o ser humano. Estes compostos sulfurados estão presentes em nosso dia-adia em diversos produtos como medicamentos, shampoo, pesticidas, conservantes alimentícios entre outros. A tiocarbamida $\left(\mathrm{NH}_{2} \mathrm{CSNH}_{2}\right)$, conhecida como tiouréia, é o principal produto da degradação dos compostos sulfurados no meio ambiente, contaminando principalmente os recursos hídricos como rios, lagos e oceanos [1]. De acordo com a resolução do Conama $357 / 2005$, os níveis de sulfetos não dissociados presentes em águas apropriadas para o consumo humano devem atingir um valor máximo de $0,002 \mathrm{mg} \mathrm{L}^{-1}$. Os sulfetos em baixa concentração podem ser metabolizados pelo corpo humano, porém, em concentrações maiores podem causar sérios danos 
ao organismo. Dentre os sintomas de uma intoxicação por sulfetos podemos destacar palpitações cardíacas, arritmias cardíacas, bronquites, edemas pulmonares, dores de cabeça, tontura, tosse e convulsões [1].

Dentre os métodos existentes para a análise de tiouréia e de outros compostos contendo grupos sulfetos em sua constituição, pode-se destacar os métodos cromatográficos, que além de apresentarem um alto custo em sua aparelhagem apresentam como desvantagens a necessidade de etapas para a amostragem e tratamentos da amostra, o que inviabiliza a utilização desses métodos para a realizações de análises em campo [2]. Os métodos espectrofotométricos, por sua vez apresentam baixa sensibilidade em relação aos métodos cromatográficos, sendo que, a sensibilidade desse método está relacionada com a propriedade óptica de cada analito e depende ainda do número de centros ópticos ativos da molécula [2]. Os métodos espectrofotométricos ainda apresentam a desvantagem de uma grande quantidade de resíduos gerados durante a realização das análises. Outra técnica utilizada para a determinação de sulfetos é a titulação potenciométrica. A principal desvantagem dessa técnica para os demais métodos citados consiste no tempo necessário para se realizar as titulações, além de necessitar de uma grande quantidade de analito para se obter resultados satisfatórios.

Nesse contexto, há a necessidade de desenvolvimento de métodos para a determinação de tiouréia que apresentem alta sensibilidade, reprodutibilidade, baixo custo, simplicidade e tempo reduzido de análise. Tais características podem ser encontradas em metodologias eletroanalíticas utilizando eletrodos modificados. De acordo com Souza [3] os eletrodos modificados adquirem as propriedades do seu agente modificador e proporcionam um controle da natureza físico-química da interface eletrodo/solução. Este controle da superfície aumenta a reatividade e a seletividade do eletrodo com o analito de interesse, permitindo assim, a produção de eletrodos para vários fins e aplicações [4]. Entre as espécies utilizadas para modificar a superfície de eletrodos, a combinação entre o íon ferricianeto e os metais de transição representam uma opção viável para a preparação de eletrodos modificados, uma vez que uma grande variedade desses compostos pode ser facilmente preparada a partir de substâncias químicas de custo reduzido. Além disso, o produto dessa combinação tem uma considerável eletrodeposição em diferentes superfícies [4]. Os trabalhos de Castro et al. [5] e Gholivand et al. [6] mostram que a combinação entre o íon ferricianeto e o íon $\mathrm{Cu}^{+2}$ resulta no complexo ferricianeto de cobre (CuHCF) que é facilmente eletrosintetizado e possui uma boa estabilidade eletroquímica. Marins [7] ainda relata que eletrodos modificados com CuHCF apresentam respostas analíticas interessantes para compostos que possuem estruturas análogas a tiouréia.

As metodologias eletroanalíticas normalmente empregam técnicas como a Potenciometria, Voltametria e a Amperometria. As principais vantagens das técnicas amperométricas em relação as outras técnicas eletroanalíticas, consiste no menor efeito de contaminação da superfície do eletrodo de trabalho devido a aplicação de um potencial específico característico da espécie de interesse. Outra vantagem deste método consiste numa maior sensibilidade, onde a presença de uma mínima corrente capacitiva (corrente existente na interface eletrodo/solução) favorece a medida de menores correntes faradáicas (corrente associada com as reações de oxirredução da espécie de interesse) [8]. Nesse âmbito, o objetivo desse trabalho consiste em desenvolver um eletrodo modificado com ferricianeto de cobre e aplicá-lo na determinação de tiouréia em meio aquoso utilizando a técnica de amperometria, visando a aplicação deste método em análises de amostras reais de águas.

\section{Materiais e Métodos}

Todos os estudos eletroquímicos foram realizados numa célula composta por três eletrodos: um eletrodo auxiliar constituído por um fio de platina, um eletrodo de referência de $\mathrm{Ag} / \mathrm{AgCl}$ saturado e um eletrodo de trabalho de carbono vítreo (GC) com 3mm de diâmetro e aproximadamente $7,1 \mathrm{~mm}^{2}$ de área. Para todos os estudos eletroquímicos utilizou-se um Potenciostato/Galvanostato AUTOLAB - PGSTAT 128N controlado pelo software GPES 4.9 conectado a um computador adequado.

Todo os reagentes utilizados neste trabalho foram de pureza analítica (PA) e todas as soluções foram preparadas utilizando água ultrapura obtida por um purificador de água MEGAPURITY - MEGA RO/UP. Antes da modificação da superfície do eletrodo de GC com ferricianeto de cobre (CuHCF), o mesmo foi submetido a sucessivos polimentos mecânicos com suspensão de alumina $0,05 \mu \mathrm{m}$ até que a sua superfície adquirisse um aspecto espelhado. Após cada polimento, o eletrodo era lavado com água ultrapura para retirar o excesso de alumina adsorvido em sua superfície.

Para a modificação da superfície do eletrodo de GC com CuHCF, o mesmo foi imerso numa solução de $1,0 \times 10^{-3} \mathrm{~mol} \mathrm{~L}^{-1}$ de $\mathrm{K}_{3} \mathrm{Fe}(\mathrm{CN})_{6}$ e numa solução de $1,0 \times 10^{-3} \mathrm{~mol} \mathrm{~L}^{-1}$ de $\mathrm{Cu}\left(\mathrm{NO}_{3}\right)_{2} \cdot 3 \mathrm{H}_{2} \mathrm{O}$, ambas preparadas numa solução eletrolítica de $\mathrm{KNO}_{3} 1,0 \times 10^{-1} \mathrm{~mol} \mathrm{~L}^{-1}$ e empregando a técnica de voltametria cíclica, aplicou-se uma faixa de potencial de 0,4 a $1,0 \mathrm{~V}$ com uma velocidade de varredura de $50 \mathrm{mV} \mathrm{s}^{-1}$ durante 40 ciclos voltamétricos. Após a formação do filme de CuHCF, o eletrodo modificado foi lavado com água ultrapura para 
a remoção de traços da solução modificadora em contato com sua superfície, visto que estes resíduos poderiam influenciar no desenvolvimento deste trabalho.

Após a preparação do eletrodo modificado foi realizado um estudo de velocidade do mesmo para diagnosticar o mecanismo das reações eletroquímicas ocorridas em sua superfície e foi verificada sua interação com a molécula de tiouréia para posteriormente realizar a sua determinação em meio aquoso através da técnica de amperometria.

\section{Resultados e Discussão}

A primeira etapa deste trabalho consistiu na modificação da superficie do eletrodo de carbono vitreo (GCE) com o complexo ferricianeto de cobre (CuHCF) utilizando a tecnica de voltametria cíclica. A Figura 1 mostra o crescimento do filme modificador de CuHCF sobre a superfície do GCE durante sucessivos ciclos voltamétricos utilizando uma faixa de potencial de 0,4 a $1,0 \mathrm{~V}$ numa velocidade de varredura de $50 \mathrm{mV} \mathrm{s}^{-1}$.

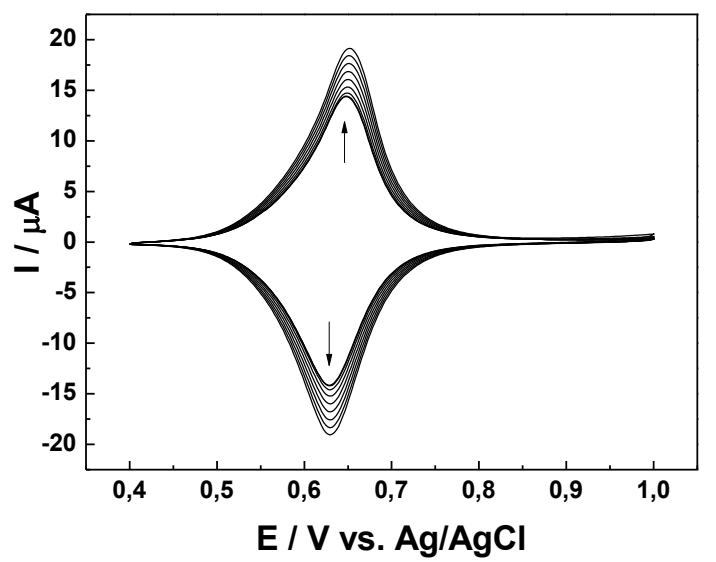

gura 1: Voltamogramas cíclicos obtidos durante a modificação do eletrodo de GC com CuHCF através da técnica de voltametria cíclica utilizando uma faixa de potencial de 0,4 a $1,0 \mathrm{~V}$ durante 40 ciclos em velocidade de varredura de $50 \mathrm{mV} \mathrm{s}^{-1}$.

$\mathrm{Na}$ Figura 1 observa-se os voltamogramas cíclicos obtidos durante a modificação do eletrodo de carbono vítreo com ferricianeto de cobre (CuHCF), onde nota-se um aumento constante das correntes de pico anódica e catódica ao final de cada ciclo de varredura, indicando o crescimento do filme de CuHCF pelo processo de eletrodeposição na superfície do eletrodo de GC. O comportamento eletroquímico do complexo eletrossintetizado possui características semelhantes àqueles descritos na literatura por Castro et al. [5] e Gholivand et al. [6], onde são observados a presença de um par de pico reversível em 0,63 V, referente aos processos redox do filme de CuHCF, conforme descrito pela reação (I):

$$
\mathrm{Cu}_{3}{ }_{3}\left[\mathrm{Fe}^{\prime \prime \prime}(\mathrm{CN})_{6}\right]_{2}+2 \mathrm{e}^{-}+2 \mathrm{~K}^{+} \leftrightarrow \mathrm{K}_{2}\left\{\mathrm{Cu}_{3}{ }_{3}\left[\mathrm{Fe} e^{\prime \prime}(\mathrm{CN})_{6}\right]_{2}\right\} \quad \text { (I) }
$$

De acordo com Mattos e Gorton [9] o processo redox do filme de CuHCF envolve a redução do $\mathrm{Fe}^{3+}$ para $\mathrm{Fe}^{2+}$ e não a redução da espécie $\mathrm{Cu}^{2+} \leftrightarrow \mathrm{Cu}^{+}$, a qual ocorre em potenciais fora da faixa de trabalho investigada. Além disso, observa-se na reação I que durante a reação de oxirredução há a incorporação dos íons $\mathrm{K}^{+}$à estrutura do filme contribuindo para a estabilidade e eletroneutralidade do complexo formado.

Com o objetivo de verificar como ocorrem os processos eletroquímicos na superfície do eletrodo modificado com CuHCF, o mesmo foi submetido em uma solução eletrolítica de $\mathrm{KNO}_{3} 1,0 \times 10^{-1} \mathrm{~mol} \mathrm{~L}^{-1}$ a diferentes ciclos voltamétricos em velocidades de varredura distintas numa faixa de potencial de 0,4 a 1,0 V. A influência da velocidade de varredura no controle dos processos eletroquímicos ocorridos na superfície do eletrodo modificado com CuHCF é apresentado pelas Figuras $2 \mathrm{~A}$ e $2 \mathrm{~B}$ :
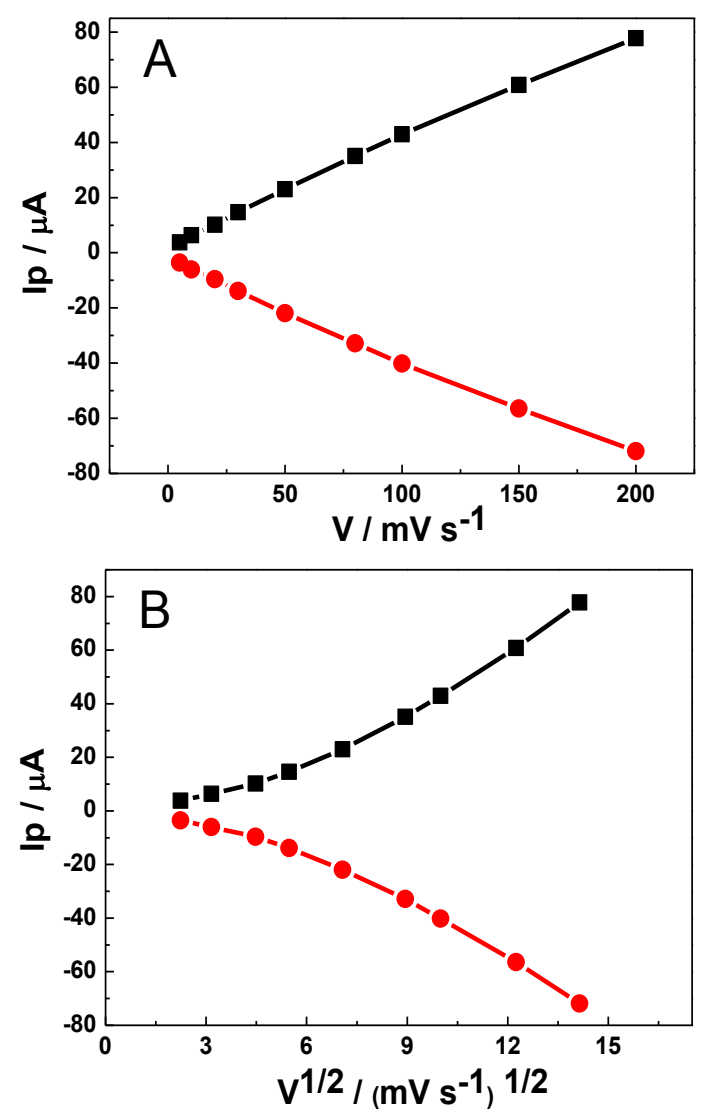

ura 2: Relação das correntes de pico anódica e catódica com (A) velocidade de varredura e $(B)$ raiz quadrada das seguintes velocidades de varredura: $5 \mathrm{mV} \mathrm{s}^{-1} ; 10 \mathrm{mV} \mathrm{s}^{-1} ; 20 \mathrm{mV} \mathrm{s}^{-1} ; 30 \mathrm{mV} \mathrm{s}^{-1} ; 50 \mathrm{mV} \mathrm{s}^{-1} ; 80$ $\mathrm{mV} \mathrm{s}^{-1} ; 100 \mathrm{mV} \mathrm{s}^{-1} ; 150 \mathrm{mV} \mathrm{s}^{-1}$ e $200 \mathrm{mV} \mathrm{s}^{-1}$.

Através deste estudo, verificou-se que as correntes de pico anódica e catódica varia linearmente com a velocidade de varredura, conforme mostra a Figura 2A, indicando que o processo de transferência de carga é controlado somente pelas reações eletroquímicas das espécies adsorvidas na superfície. Além disso, observa-se na Figura 2B que a variação das correntes de pico anódica e catódica não apresenta um 
comportamento linear em função da raiz quadrada da velocidade de varredura, indicando que não há um controle difusional no transporte de carga na superfície do filme. Desta forma, o controle da transferência de carga durante as reações de oxi-redução ocorre somente pelas espécies adsorvidas na superfície do eletrodo [6].

A etapa seguinte deste trabalho consistiu na verificação do comportamento eletroquímico do eletrodo modificado com CuHCF na presença da molécula de tiouréia $\left(\mathrm{NH}_{2} \mathrm{CSNH}_{2}\right)$. Nessa etapa, para fins comparativos, inicialmente obteve-se apenas o perfil ciclovoltamétrico da molécula tiouréia $\left(10 \times 10^{-3} \mathrm{~mol} \mathrm{~L}^{-1}\right)$ sobre a superfície do GCE convencional utilizando uma faixa de potencial de 0,4 a 1,0 V numa velocidade de varredura de $20 \mathrm{mV} \mathrm{s}^{-1}$. Posteriormente, analisou-se 0 comportamento eletroquímico do eletrodo modificado com CuHCF na presença de uma solução de tiouréia $10 \times 10^{-3} \mathrm{~mol} \mathrm{~L}^{-1}$ preparada numa solução eletrolítica de $\mathrm{KNO}_{3} 1,0 \times 10^{-1} \mathrm{~mol} \mathrm{~L}^{-1}$ empregando-se uma faixa de potencial de 0,4 a $1,0 \mathrm{~V}$ numa velocidade de varredura de $20 \mathrm{mV} \mathrm{s}^{-1}$. Os resultados obtidos com estes estudos são apresentados pelas Figuras 3 e 4, respectivamente.

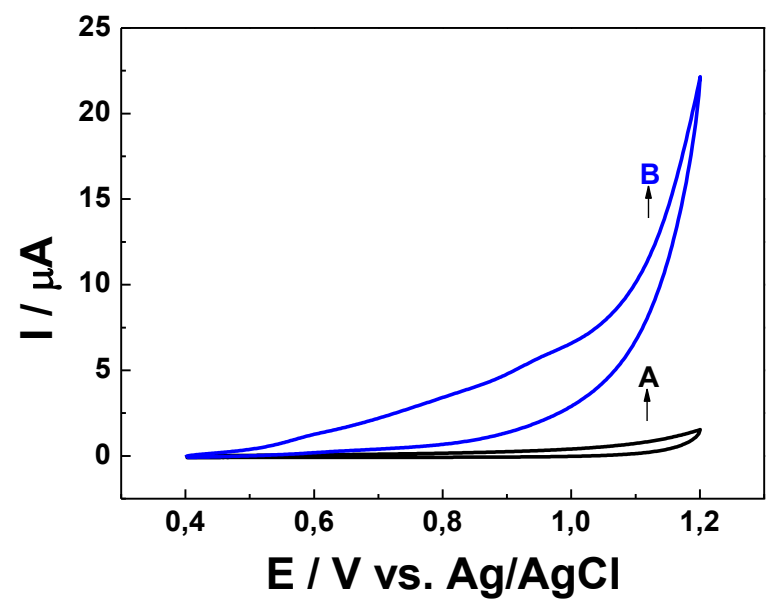

Figura 3: Perfil voltamétrico do GCE convencional na (A) ausência e na (B) presença de uma solução de tiouréia $10 \times 10^{-3} \mathrm{~mol} \mathrm{~L}^{-1}$.

A Figura 4 apresenta o perfil voltamétrico do eletrodo modificado com CuHCF na ausência e na presença da solução de tiouréia $10 \times 10^{-3} \mathrm{~mol} \mathrm{~L}^{-1}$. Ao analisar esta figura, observa-se que 0 voltamograma $A$ correspondente ao eletrodo modificado com CuHCF na presença de uma solução eletrolítica de $\mathrm{KNO}_{3} 1,0 \times 10^{-1}$ $\mathrm{mol} \mathrm{L}^{-1}$, onde o mesmo apresentou seu comportamento característico para as condições empregadas.

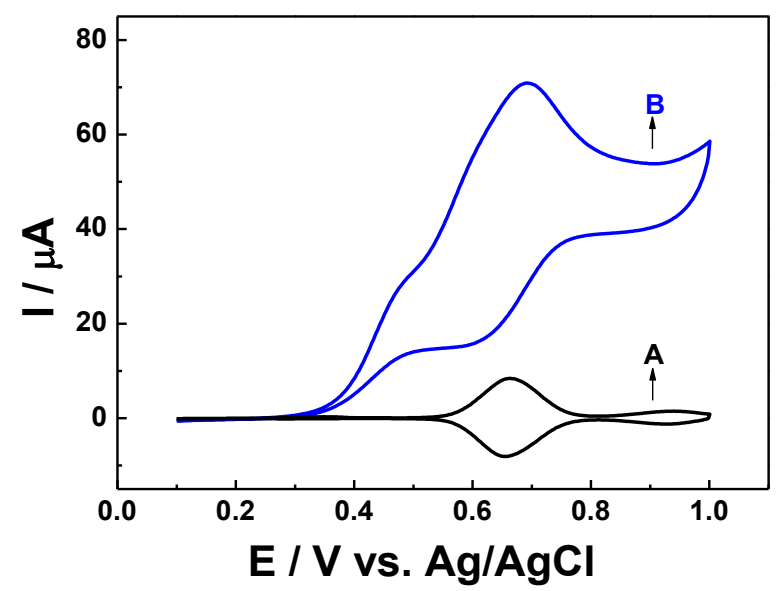

Figura 4: Perfil voltamétrico do eletrodo modificado com CuHCF na (A) ausência e na (B) presença de uma solução de tiouréia $10 \times 10^{-3} \mathrm{~mol} \mathrm{~L}^{-1}$.

O voltamograma B corresponde ao eletrodo modificado com CuHCF em presença da solução de tiouréia $10 \times 10^{-3} \mathrm{~mol} \mathrm{~L}^{-1}$, onde verifica-se que o filme de CuHCF apresentou um potencial de pico anódico em aproximadamente $0,69 \mathrm{~V}$ referente a eletrooxidação da molécula de tiouréia. Já a corrente de pico catódica apresentou uma diminuição em sua intensidade acompanhado por um leve deslocamento desta para potencias mais negativos.

De acordo com Bolzán et al. [10], a eletrooxidação da tiouréia em soluções eletrolíticas contendo íons nitrato promove a formação da espécie dissulfeto de formamidina desprotonado. Em soluções eletrolíticas ácidas, o par redox envolvendo a tiouréia e o dissulfeto de formamidina é descrito pela reação (III):

$$
2 \mathrm{NH}_{2} \mathrm{CSNH}_{2} \leftrightarrow\left[\left(\mathrm{H}_{2} \mathrm{~N}\right)_{2}\left(\mathrm{H}_{2} \mathrm{~N}\right)_{2} \mathrm{C}_{2} \mathrm{~S}_{2}\right]^{2+}+2 \mathrm{e}^{-} \quad \text { (III) }
$$

Após a verificação da interação entre o eletrodo modificado com a molécula de tiouréia, foi realizada a construção da curva analítica através da técnica de amperometria num intervalo de concentração de $5 \times 10^{-4}$ a $30 \times 10^{-4} \mathrm{~mol} \mathrm{~L}^{-1}$ utilizando um potencial fixo de $0,69 \mathrm{~V}$. O incremento de corrente adquirido após cada adição de uma determinada concentração de tiouréia pode ser observado pelo amperograma mostrado na Figura 5 e a curva analítica obtida através desta técnica pode ser visualizada pela Figura 6. 


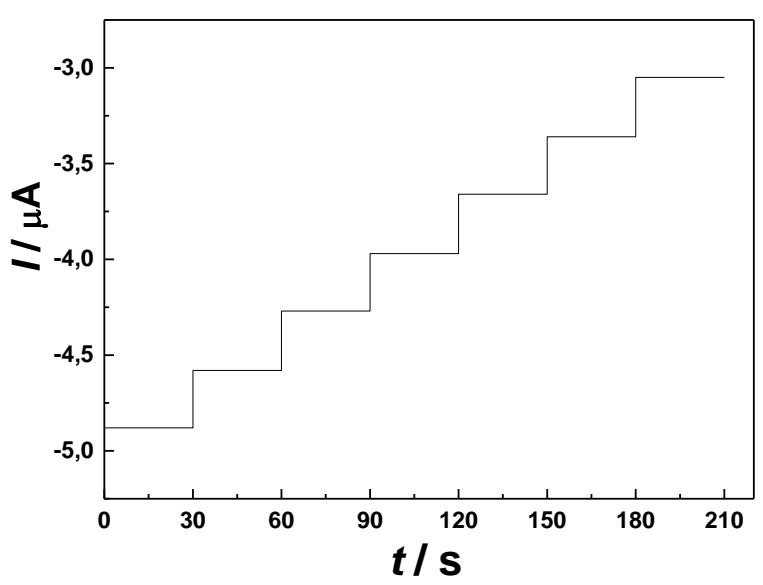

Figura 5: Amperograma obtido para o eletrodo modificado com CuHCF através de sucessivas adições de tiouréia num intervalo de $5 \times 10^{-4} \mathrm{a}$ $30 \times 10^{-4} \mathrm{~mol} \mathrm{~L}^{-1}$, em potencial fixo de $0,69 \mathrm{~V}$.

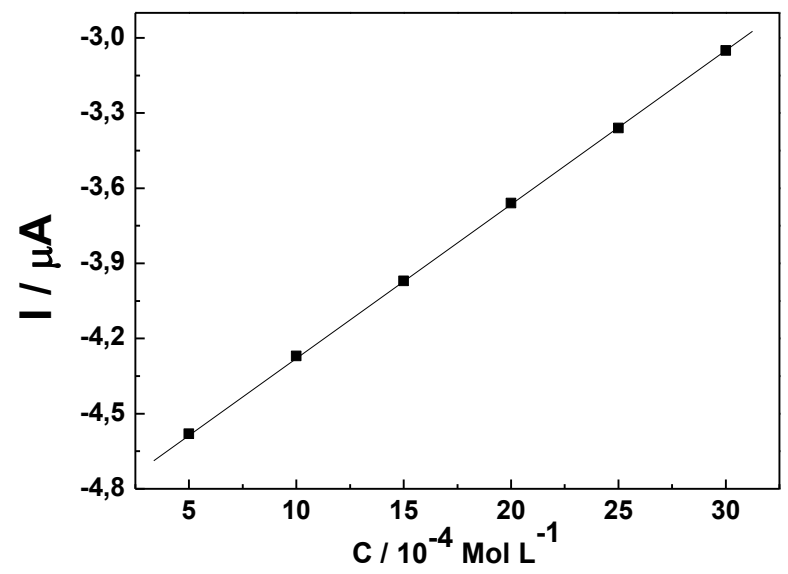

Figura 6: Curva analítica obtida para o eletrodo modificado com CuHCF utilizando as concentrações de $5 ; 10 ; 15 ; 20 ; 25$ e $30 \times 10^{-4} \mathrm{~mol} \mathrm{~L}^{-1}$ de tiouréia.

Analisando a curva analítica apresentada pela Figura 6, observa-se que o método desenvolvido apresenta uma faixa linear entre $5 \times 10^{-4}$ e $30 \times 10^{-4} \mathrm{~mol} \mathrm{~L}^{-1}$ com uma equação de regressão expressa por $\mathrm{I}_{\mathrm{pa}}(\mathrm{A})=6,10857 \times 10^{-4} \mathrm{c}\left(\mathrm{mol} \mathrm{L}^{-1}\right)-4,884 \times 10^{-6}$, um coeficiente de correlação $(r)$ de 0,99997 e um limite de detecção (LOD) de $1,6 \times 10^{-5} \mathrm{~mol} \mathrm{~L}^{-1}$. A relação linear observada na Figura 6 entre os valores de corrente obtidos vs. as concentrações de tiouréia indicam uma possibilidade promissora de utilização deste eletrodo para a determinação de tiouréia em amostras reais de água.

\section{Conclusões}

O emprego da técnica de voltametria cíclica neste trabalho possibilitou a modificação de um eletrodo de GC com um filme de CuHCF e a verificação da interação deste eletrodo modificado com a molécula de tiouréia. A resposta linear obtida por este dispositivo, através da técnica de amperometria, demostra a viabilidade de sua utilização para a detecção de tiouréia em amostras reais de água, visto que este composto reflete o nível de contaminação das águas por compostos sulfurados, que por sua vez são bioacumulativos e tóxicos para o ser humano.

\section{Agradecimentos}

Os autores agradecem à Fundação de Amparo à Pesquisa do Espírito Santo (FAPES) e ao Programa Institucional de Iniciação Cientifica (PIIC) da Universidade Federal do Espirito Santo.

\section{Referências}

[1] MATIAS, A.E.B. Desenvolvimento e caracterização de sensores quimicamente modificados para análises de sulfeto. 2010. 71f. Dissertação (Mestrado em Engenharia do Meio Ambiente - Recursos Hídricos e Saneamento Ambiental) - Universidade Federal de Goiás, Goiás, 2010.

[2] PETRUCI, J.F.S. Sensores ópticos para quantificação de sulfeto de hidrogênio em matrizes gasosas por Espectrometria de Fluorescência no UVvisível e Espectroscopia de absorção no Infravermelho, 2014,103f. Tese (Doutorado em Química) Instituto de Química, Universidade Estadual Paulista, Araraquara, 2014.

[3] SOUZA, M.F.B. Quím. Nova. V.20, p.191-195, 1996.

[4] POURNAGHI-AZAR, M.H.; AHOUR, F. J. Elctroanal. Chem. V.622, p.22-28, 2008.

[5] - CASTRO, S.S.L.; OLIVEIRA, M.F.; STRADIOTTO, N.R. Int. J. Electrochem. Sci., V.5, p.1447 - 1456, 2010.

[6] GHOLIVAND, M.G.; KHODADADIAN, M.; OMIDI, M. Materials Science and Engineering C. V.33, p.774-781, 2013.

[7] MARINS, D.M.S. Desenvolvimento de eletrodos modificados para determinação de compostos sulfurados em gasolina, 2008, 166f. Tese (Doutorado em Química). Instituto de Química, Universidade Estadual Paulista, Araraquara, 2008.

[8] SANTOS, W.T.P.; GIMENES, D. T.; RICHTER, E. M.; ANGNES, L. Quím. Nova, v.34, p.1753-1761, 2011.

[9] MATTOS, I.L.; GORTON, L. Quim. Nova, V. 24, p.200-205, 2001.

[10] BOLZÁN, A.E.; WAKENGE, I.B.; SALVAREZZA, R.C.; ARVIA, A.J. J. Electroanal. Chem., V.475, p.181-189, 1999. 